\title{
Nonreciprocal diffraction through dielectric gratings with two-dimensional chirality
}

\author{
A. Potts, ${ }^{*}$ W. Zhang, and D. M. Bagnall \\ School of Electronics and Computer Science, University of Southampton, Southampton SO17 1BJ, United Kingdom
}

(Received 14 August 2007; published 10 April 2008)

\begin{abstract}
It is generally assumed that the propagation of light through a dielectric medium is always reciprocal under reversal of the propagation direction. We show that in an all-dielectric diffractive system nonreciprocal polarization changes are possible for individual diffracted beams when the diffractive medium possesses twodimensional chirality. This nonreciprocity is characterized by different eigenstates for the system for the forward and reverse directions respectively, yet it is also entirely consistent with the predictions of the Lorentz reciprocity lemma.
\end{abstract}

DOI: 10.1103/PhysRevA.77.043816

PACS number(s): 42.79.Dj, 78.67.-n, 78.20.Ek, 42.81.Gs

\section{INTRODUCTION}

Nonreciprocal behavior is a much sought property in optics because it would facilitate the implementation of new forms of unidirectional optical devices. To date the only known example of nonreciprocal behavior is that arising from the Faraday effect and the associated light-matter interaction with magnetic materials.

The Faraday effect arises from the application of a dc magnetic field to an optically transparent material to generate optical birefringence within the material for the two opposite forms of circularly polarized light. The net effect of this birefringence is to produce a rotation of the plane of polarization of linearly polarized light as it propagates through the material in the direction of the dc magnetic field. This rotation of the polarization azimuth direction $\phi$ is similar to that experienced by an identical linearly polarized beam when it propagates through a material with three-dimensional (3D) chirality, such as a quartz crystal or a sugar solution. The principal difference between the two situations is that while the 3D chiral medium is reciprocal (the direction of the polarization rotation is the same for beams traveling in opposite directions), the Faraday effect is nonreciprocal.

Light beams traveling in opposite directions through a Faraday device see the dc magnetic field as acting in opposite directions. Consequently, they also experience a reversal of the circular birefringence in the optically transparent material upon which the magnetic field acts due to an interchanging of the refractive indices for the two opposite circularly polarized eigenstates. This results in a reversal of the rotation direction for the azimuth of linearly polarized light. The Faraday device is therefore equivalent to a chiral material in which the sense of chirality is reversed when viewed from opposite directions. Such a reversal of chiral handedness is also a characteristic of the geometric property of $2 \mathrm{D}$ chirality [1]. This has therefore led to speculation over whether materials or structures that exhibit $2 \mathrm{D}$ chirality at a given length-scale could exhibit optical activity or dichroism in some form [2].

Until recently it was assumed that materials that possess 2D chirality in either their surface topology or their molecu-

\footnotetext{
*ap@ecs.soton.ac.uk
}

lar arrangement were incapable of exhibiting optical activity. However, we recently demonstrated that some thin-film structures patterned with periodic square arrays of diffracting elements can strongly affect the polarization state of optical beams diffracted from their surface, provided those chiral elements exhibit 2D chirality in their surface topology, and their periodicity is larger in scale than the wavelength of the incident electromagnetic radiation. These structures we have collectively termed planar chiral materials (PCMs). As the periodicity of the surface patterning in these structures is greater than the wavelength of the incident radiation we use to characterize their optical properties, and the structures therefore generate well defined rectangular diffraction patterns, we will refer to these structures as diffractive PCMs.

Initially our research on diffractive PCMs concentrated on the study of chirally patterned metallic films $[3,4]$. These structures were the first to demonstrate the capability to rotate and elliptize the polarization state of light diffracted from their surface in a manner sensitive to both the handedness and the magnitude of the surface chirality [3,4], and the chirality-dependent polarization changes were even seen when the input polarization state was parallel to the axes of the diffraction gratings, thereby distinguishing the behavior of these structures from that seen previously for achiral gratings $[5,6]$. However, most of our metallic chiral films were fabricated on substrates of high resistivity silicon that are only transparent for wavelengths above $1.2 \mu \mathrm{m}$ (i.e., in the infrared). Consequently all investigations of their chiral properties in the visible region of the electromagnetic spectrum had to be carried out in reflection. This, though, negates the primary advantage that planar chiral structures have with regard to their more familiar three-dimensional variants: the reversal of their sense of chirality for opposite directions of electromagnetic wave propagation. It is this latter feature that makes these structures so intriguing as it offers the possibility of designing materials with optical properties that may be nonreciprocal or nonreversible under certain circumstances or for individual diffracted beams. It was therefore crucial that this area of research was extended to include optically transparent structures so that evidence of nonreciprocity could be sought.

Subsequent to our work on diffractive metallic PCMs, Kuwata-Gonokami et al. [7] reported on the gyrotropy of light transmitted through arrays of gold gammadions on optically transparent quartz substrates. Their results indicated 
that the directly transmitted beam could exhibit a polarization change, but that this optical activity was reciprocal (i.e., the same for opposite directions of light propagation). They therefore concluded that the polarization change from their structures was induced by 3D chirality that arose from the interaction of the 2D chiral surface patterning of the metallic surface layer in the $x y$ plane with an asymmetry in the order in which the different material layers of the sample were arranged in the perpendicular $z$ direction. However, while they omitted to investigate the optical properties of nonzero order diffracted beams, their results for the zero-order beam raised concerns over whether the polarization changes we observed for diffractive PCMs in reflection $[3,4]$ could also be a consequence of 3D chirality.

In order to determine the dimensionality of the chirality responsible for the polarization effects seen for PCMs, we extended our investigations to include 2D chiral structures constructed entirely from dielectric materials such as silicon nitride and silicon dioxide [8]. These all-dielectric PCMs are also seen to exhibit a chirality-specific optical activity that is similar in form to that previously reported for metallic PCM gratings [3,4], and this occurs despite these all-dielectric PCMs containing none of the induced currents and surface plasmons that have been proposed as potential mechanisms for the optical activity in metallic PCMs. This suggests that another mechanism may be applicable in this case. One possibility is interference effects [8].

In this paper we report the results of a series of polarization experiments performed at $632.8 \mathrm{~nm}$ in transmission on these dielectric PCMs. These experiments clearly demonstrate that the polarization changes manifested by light diffracted through these structures are nonreciprocal. Replacing a PCM sample with its enantiomer results in a reversal of the optical activity, but so too does reversing the direction of light propagation through the same sample. As both of these processes result in a reversal of the $2 \mathrm{D}$ chirality of the structure (as seen by the incident beam), this clearly demonstrates that the chiral effect is $2 \mathrm{D}$ in character and not $3 \mathrm{D}$. We also show that the different polarization changes for structures of different 2D chirality are also characterized by differing eigenstates in each case and different polarization-dependent transmission coefficients. By considering the electromagnetic field distribution for two opposing propagation directions in detail we show that this optical activity, while being nonreciprocal in character, is entirely consistent with the Lorentz reciprocity lemma $[9,10]$. Although the small polarization changes reported by Kuwata-Gonokami et al. [7] for the zero-order transmitted beams are probably due to 3D chirality, our experiments indicate that the much larger polarization changes seen for higher order diffracted beams are almost entirely the result of 2D chirality and, consequently, are nonreciprocal in behavior.

\section{EXPERIMENTAL DETAILS}

The planar chiral material (PCM) samples studied in this paper all consisted of planar chiral patterns that were etched through $\mathrm{Si}_{3} \mathrm{~N}_{4}$ layers of various thicknesses that were supported on 1-mm-thick fused silica (quartz) wafers. Fused

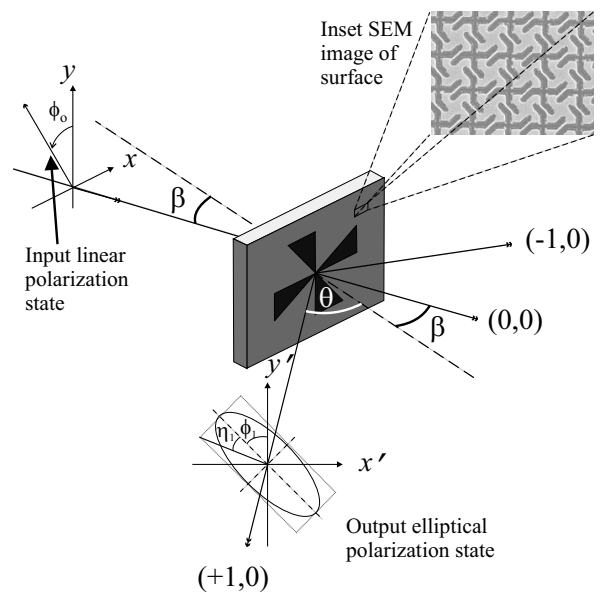

FIG. 1. A schematic illustration of the experimental setup used to optically characterize the planar chiral structures. The polarization state of the output diffracted beam is defined by its polarization azimuth $\left(\phi_{1}\right)$ and ellipticity $\left(\eta_{1}\right)$, with $\phi_{1}$ being measured in an anticlockwise direction from the vertical as viewed by an observer looking directly into the beam. $\phi_{0}$ and $\eta_{0}$ are the equivalent terms for the incident beam. The angle of incidence $(\beta)$ and the diffraction angle $(\theta)$ are measured with respect to the sample normal as indicated. The large black cross on the sample surface is depicted to represent the fourfold symmetry of the sample surface and its sense of chirality as viewed from this direction of observation. It is not a real feature of the sample. Also shown (inset) is a high-resolution micrograph of an actual sample showing part of the array of gammadions. In this sample each of the four arms of the gammadion has a bending angle $\alpha=-45^{\circ}$.

silica was chosen because it is transparent at $632.8 \mathrm{~nm}$ while the fused structure ensured that the substrates were neither optically active nor birefringent, unlike single-crystal quartz, which can be both.

The samples were manufactured using standard microfabrication techniques as described previously [8]. The first step was the deposition of a layer of $\mathrm{Si}_{3} \mathrm{~N}_{4}$ onto one surface of a fused silica wafer by plasma-enhanced chemical vapor deposition (PECVD). This was then patterned with the different planar chiral designs using a combination of electron beam lithography and reactive ion etching $[8,11]$. Each chiral design consisted of a square array of gammadions in a planar tiling arrangement (of type 442 in the Orbifold notation of wallpaper group symmetries or $\mathrm{p} 4$ in the International Union of Crystallography notation) with an identical pitch $(\Lambda$ $=5.0 \mu \mathrm{m})$ in both the vertical and horizontal directions of the array. The area of each array was approximately $1 \mathrm{~mm}^{2}$. Each individual gammadion consisted of four identical arms with the angle between each pair of adjacent arms being $90^{\circ}$, while each gammadion arm was comprised of two distinct line segments of equal length $(\xi=1.8 \mu \mathrm{m})$ with the angle between the two segments being $\pm 135^{\circ}$ (see Fig. 1, inset). This corresponds to an arm bending angle $(\alpha)$ of $\pm 45^{\circ}$. Each gammadion therefore possesses fourfold rotational symmetry about its center, but no line of mirror symmetry, while the gammadion arrays also possess fourfold rotational symmetry, as well as translational symmetry in both lattice directions. Consequently they also possess no birefringence in the plane 
of the sample, but do exhibit 2D chirality. A more comprehensive account of the fabrication process and of the gammadion design parameters $(\alpha, \xi$, and $\Lambda)$ has been reported previously $[8,11]$.

Optical experiments were then performed to determine the polarization altering properties of the PCMs. This involved measuring the output polarization state for light diffracted from the various chiral gammadion arrays for different input polarization states, and calculating the polarization change in each case (see Fig. 1 for a schematic diagram of the experimental arrangement). The polarization states of these beams were measured by the rotating wave plate polarimetric method using a commercial polarimeter system (Thorlabs model \#PA510-EC) with a wavelength range of 450 to $700 \mathrm{~nm}$. The light source used was a HeNe laser operating at $632.8 \mathrm{~nm}$ that first passes through a linear polarizer and then through a Fresnel rhomb prism in order to generate a circularly polarized beam. A second linear polarizer is then used to convert the beam back into a linearly polarized state, the azimuthal angle of which $\left(\phi_{0}\right)$ can be rotated radially about the beam direction by rotating the second polarizer. This experimental arrangement therefore allows the direction of linear polarization of the incident beam to be rotated about the propagation direction without changing the beam's intensity. Finally, the beam is focused onto a single gammadion array using a long focal length planoconvex lens.

After the incident beam hits the array of chiral gammadions, diffracted waves propagate at various angles, creating a well-defined rectangular diffraction pattern. The sample itself is then rotated about its normal until the two perpendicular axes of the square array of chiral elements are parallel to, and perpendicular to, the plane of the optical bench, respectively. The horizontal plane that includes the zero-order beam we then define to be the plane of incidence as it is the plane that includes both the input and directly reflected beams, as well as the normal to the sample. The two first-order beams that lie either side of the zero-order beam in this plane we denote as the $(+1,0)$ and $(-1,0)$ orders, respectively.

In order to determine the presence of any non-reciprocal effects in the optical properties of these PCM structures we have studied various pairs of enantiomeric samples that are related by mirror (or parity) inversion. Each sample consisted of a square array of gammadions with an arm bending angle of either $\alpha=-45^{\circ}$ (left-handed gammadions) or $\alpha=$ $+45^{\circ}$ (right-handed gammadions). The pitch $(\Lambda=5.0 \mu \mathrm{m})$ and arm segment length $(\xi=1.8 \mu \mathrm{m})$ were the same for all samples. However, the thickness $(t)$ of the $\mathrm{Si}_{3} \mathrm{~N}_{4}$ film into which each array of gammadion-shaped holes was etched was varied from sample to sample, with $t$ ranging from 80 $\mathrm{nm}$ for samples W5BA42 and W5BA45 to $320 \mathrm{~nm}$ for samples W3BA42 and W3BA45.

In order to investigate the principle of reciprocity in these nonmagnetic diffractive planar chiral media, we have restricted ourselves to the study of the polarization parameters of the two first-order diffracted beams in the plane of incidence only. These beams were chosen partly because of their relatively large luminous intensities and their proximity to the zero-order beam (the angle of diffraction being less than $8^{\circ}$ ). The zero-order beam itself was not investigated thor- oughly because it, uniquely among all the diffracted beams, consistently failed to exhibit any polarization change relative to the incident beam. This result is somewhat different from that reported by Kuwata-Gonokami et al. [7], but not necessarily contradictory.

The polarization changes reported by Kuwata-Gonokami et al. for transmission of the zero-order beam through PCMs of gold gammadions on quartz [7] were more than an order of magnitude smaller than those we have reported for the first-order beams diffracted from various PCM structures $[4,8,12]$. They were also reciprocal, and were therefore attributed to 3D chiral effects arising from an interaction of the 2D chirality of the surface topology and the asymmetric layer structure [7]. In the next section we will show using the Lorentz reciprocity lemma that 2D chirality on its own cannot give rise to optical activity in the zero-order beam at normal incidence. Thus any polarization changes that are detected in the zero-order beam for systems with rotational symmetry but no birefringence must be due to 3D chirality.

The reason for the absence of any optical activity in our dielectric PCM samples due to 3D chiral effects of the type reported by Kuwata-Gonokami et al. [7] is still unclear. It may be that such effects are indeed present, but that they are even smaller in magnitude than those reported by KuwataGonokami et al. [7] and, consequently, cannot be detected with our experimental arrangement. Alternatively, the physical mechanism responsible for such effects may be dependent on the material composition of the sample, and may require metallic components in order to become evident.

The first-order diffracted beams on the other hand have already been shown to be capable of exhibiting strong chirality-dependent polarization changes [8]. In Sec. IV the nonreciprocal properties of these diffractive dielectric PCM samples will be demonstrated by examining the polarization changes of the first-order transmitted beams when the chirality of the sample, its orientation and the beam propagation direction are all reversed independently. Nevertheless, these results do not violate the Lorentz reciprocity lemma that is discussed in the next section.

\section{THEORY OF RECIPROCITY}

In the fields of optics and electromagnetism the concept of reciprocity refers to a variety of related theorems that describe the behavior of linear optical systems under the interchange of sources and detectors, or the spatial inversion of the system. One such example is that of the Helmholtz reciprocity theorem. This is often applied to simple optical systems in which the light from an object is either, reflected, refracted or diffracted by a mirror, a lens, an aperture or a diffraction grating [see Fig. 2(a)]. The amplitude of the light wave at any given point in such systems can be determined via the Fresnel-Kirchhoff diffraction formula [13]

$$
U(Q)=-\frac{i A}{2 \lambda} \oint_{S} \frac{e^{\mathrm{i} \cdot \mathbf{k}(\mathbf{r}+\mathbf{s})}}{r s} I(\mathbf{R})\left[\frac{\mathbf{n} \cdot \mathbf{r}}{r}-\frac{\mathbf{n} \cdot \mathbf{s}}{s}\right] d S .
$$

This relates the relative amplitude and phase $U(Q)$ of an optical disturbance at a point $Q$ (the image) to that of the 

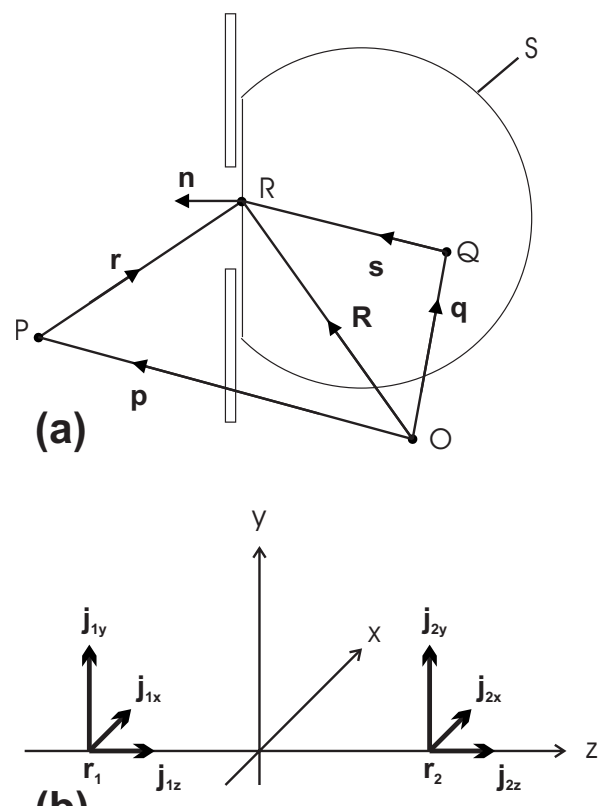

(b)

FIG. 2. (a) An illustration of the vectors used to define the Fresnel-Kirchhoff diffraction formula. (b) A schematic illustration of a pair of current sources used to verify Eq. (3).

original disturbance at the point $P$ that created it (the source object). The integral is performed over a closed surface $S$, defined by a vector function $\mathbf{R}$, for which $\mathbf{n}$ is the surface normal and $I(\mathbf{R})$ is the transparency of the surface (or aperture function). As Eq. (1) is symmetric under an interchange of the source and detection points $(P$ and $Q)$, the system is said to be reciprocal. Thus, if $Q$ represents the image of a source at $P$, then $P$ can also be considered to be the image of $Q$. Helmholtz reciprocity is, however, a scalar theorem and therefore cannot adequately describe electromagnetic (EM) systems where the polarization states of the various EM waves are important. For those particular situations, a second and more powerful reciprocity theorem is required: Lorentz reciprocity.

Lorentz reciprocity is a more powerful reciprocity theorem when considering polarized waves as it describes the interaction of the actual electric $\mathbf{E}(\mathbf{r})$ and magnetic $\mathbf{H}(\mathbf{r})$ field vectors for two distinct sources in a given system $[9,10]$. The theorem states that for a system with two current source distributions $\mathbf{j}_{1}(\mathbf{r})$ and $\mathbf{j}_{2}(\mathbf{r})$, their associated field distributions $\left[\mathbf{E}_{1}(\mathbf{r}), \mathbf{H}_{1}(\mathbf{r})\right]$ and $\left[\mathbf{E}_{2}(\mathbf{r}), \mathbf{H}_{2}(\mathbf{r})\right]$ will be related by the integral equation

$$
\int_{V}\left(\mathbf{j}_{1} \cdot \mathbf{E}_{2}-\mathbf{j}_{2} \cdot \mathbf{E}_{1}\right) d V=\oint_{S}\left(\mathbf{E}_{1} \times \mathbf{H}_{2}-\mathbf{E}_{2} \times \mathbf{H}_{1}\right) \cdot d \mathbf{S},
$$

where $S$ is any arbitrary closed surface of volume $V$. The general form of the reciprocity relation in Eq. (2) can be simplified for a number of special cases.

For example, if the system is an isolated one in which the surface $S$ encloses all the current sources $\mathbf{j}_{1}(\mathbf{r})$ and $\mathbf{j}_{2}(\mathbf{r})$ and all the fields within $S$ are a product only of those current sources and are not the result of externally applied fields, then it can be shown by the application of symmetry arguments that the following identity must hold:

$$
\mathbf{j}_{1} \cdot \mathbf{E}_{2}-\mathbf{j}_{2} \cdot \mathbf{E}_{1}=0 .
$$

This can be seen most clearly by considering two current elements $\mathbf{j}_{1}$ and $\mathbf{j}_{2}$ at positions $\mathbf{r}_{1}$ and $\mathbf{r}_{2}$, respectively, and resolving both into their respective Cartesian components $\left(\mathbf{j}_{1 x}, \mathbf{j}_{1 y}, \mathbf{j}_{1 z}\right)$ and $\left(\mathbf{j}_{2 x}, \mathbf{j}_{2 y}, \mathbf{j}_{2 z}\right)$, where the $Z$ direction is defined so that the $Z$ components of the two current elements are coaxial and their $X$ components are parallel, as shown in Fig. 2(b). Each of these six current elements will have its own field distribution $\left[\mathbf{E}_{1 x}(\mathbf{r})\right.$, etc.], and there will then be nine different interactions of the type defined in Eq. (3). However, all interactions between orthogonal current elements will result in zero contributions to Eq. (3) because each current element effectively acts like an electric dipole. Thus, for the interaction between $\mathbf{j}_{1 x}$ and $\mathbf{j}_{2 y}$

$$
0=\mathbf{j}_{1 x} \cdot \mathbf{E}_{2 y}\left(\mathbf{r}_{1}\right)=\mathbf{j}_{2 y} \cdot \mathbf{E}_{1 x}\left(\mathbf{r}_{2}\right) .
$$

Identical relations will hold for the other five orthogonal interactions. Only the three parallel interactions will result in nonzero scalar products, however, symmetry considerations necessitate that these contributions should cancel. For example, if the interaction between $\mathbf{j}_{1 x}$ and $\mathbf{j}_{2 x}$ has the value $\mathbf{j}_{1 x} \cdot \mathbf{E}_{2 x}\left(\mathbf{r}_{1}\right)-\mathbf{j}_{2 x} \cdot \mathbf{E}_{1 x}\left(\mathbf{r}_{2}\right)=c$, then interchanging the positions of $\mathbf{j}_{1 x}$ and $\mathbf{j}_{2 x}$ will yield $\mathbf{j}_{2 x} \cdot \mathbf{E}_{1 x}\left(\mathbf{r}_{1}\right)-\mathbf{j}_{1 x} \cdot \mathbf{E}_{2 x}\left(\mathbf{r}_{2}\right)=-c$, yet the two scenarios are identical; only the coordinate frame has been rotated. Therefore $c$ must be zero and $\mathbf{j}_{1 x} \cdot \mathbf{E}_{2 x}\left(\mathbf{r}_{1}\right)$ $=\mathbf{j}_{2 x} \cdot \mathbf{E}_{1 x}\left(\mathbf{r}_{2}\right)$. Similar arguments can be applied to the interaction of $\mathbf{j}_{1 y}$ and $\mathbf{j}_{2 y}$, and $\mathbf{j}_{1 z}$ and $\mathbf{j}_{2 z}$. Hence Eq. (3) is proven. This result is generally known as the Rayleigh-Carson reciprocity theorem and has important applications in radiative dipole scattering and antennae systems.

One consequence of Eq. (3) is that the following relation should also hold for any isolated system

$$
\oint_{S}\left(\mathbf{E}_{1} \times \mathbf{H}_{2}-\mathbf{E}_{2} \times \mathbf{H}_{1}\right) \cdot d \mathbf{S}=0 .
$$

This will also be true if the closed surface $S$ does not enclose any of the radiation sources, and the fields in $S$ all arise from external sources. It is this particular variant of the reciprocity theorem that is relevant to our particular experimental arrangement, and which we therefore need to consider in detail. This is particularly true given that the validity of Eq. (2) [and hence also of Eq. (5)] is not universal and is dependent on the material properties of the system. For example, a principal exception to the Lorentz reciprocity theorem is that of a system composed of magneto-optic materials that exhibits the Faraday effect. In such systems a violation of Lorentz reciprocity results because the application of a magnetic field to the system results in the dielectric tensor becoming asymmetric under spatial inversion. Thus, it is clear that if we are to test our planar chiral structures for nonreciprocal effects, we need to identify how such violations of reciprocity may arise and be clear about the origins of and constraints on Eq. (2). 
The starting point for the Lorentz reciprocity theorem is the vector identity

$$
\int_{V}[(\boldsymbol{\nabla} \times \mathbf{F}) \cdot \mathbf{G}-(\boldsymbol{\nabla} \times \mathbf{G}) \cdot \mathbf{F}] d V=\oint_{S}(\mathbf{F} \times \mathbf{G}) \cdot d \mathbf{S}
$$

which relates any two vector fields $\mathbf{F}(\mathbf{r})$ and $\mathbf{G}(\mathbf{r})$ over any closed surface $S$ of volume $V$. This is used as the basis for two further equations by first making the substitutions $\mathbf{F}$ $=\mathbf{E}_{1}$ and $\mathbf{G}=\mathbf{H}_{2}$, and then the substitutions $\mathbf{F}=\mathbf{E}_{2}$ and $\mathbf{G}$ $=\mathbf{H}_{1}$. After invoking Maxwell's equations to replace the $\boldsymbol{\nabla}$ $\times \mathbf{E}$ and $\boldsymbol{\nabla} \times \mathbf{H}$ terms, the two equations are subtracted to yield

$$
\int_{V}\left(\mathbf{j}_{1} \cdot \mathbf{E}_{2}-\mathbf{j}_{2} \cdot \mathbf{E}_{1}\right) d V+W=\oint_{S}\left(\mathbf{E}_{1} \times \mathbf{H}_{2}-\mathbf{E}_{2} \times \mathbf{H}_{1}\right) \cdot d \mathbf{S},
$$

where the term $W$ is given by

$$
W=\int_{V}\left\{\mathbf{H}_{1} \cdot \frac{\partial \mathbf{B}_{2}}{\partial t}+\mathbf{E}_{2} \cdot \frac{\partial \mathbf{D}_{1}}{\partial t}-\mathbf{H}_{2} \cdot \frac{\partial \mathbf{B}_{1}}{\partial t}-\mathbf{E}_{1} \cdot \frac{\partial \mathbf{D}_{2}}{\partial t}\right\} d V .
$$

It is only if $W=0$ that Eq. (7) reduces to the familiar form for the Lorentz reciprocity theorem given in Eq. (2). However, this can only be true if the material constants $\epsilon$ (permitivity) and $\mu$ (permeability), are the same for the two field distributions at any given point within the surface $S$. As the PCM structures that we are considering herein are composed entirely of quartz and $\mathrm{Si}_{3} \mathrm{~N}_{4}$, then the constraint $W=0$ should hold true. However, the experimental arrangement we will use to investigate nonreciprocal effects in dielectric PCM structures does not include any current sources within the local environment of the PCM and involves illuminating the system with an external source of monochromatic planewave radiation (i.e., a $\mathrm{HeNe}$ laser operating at $632.8 \mathrm{~nm}$ ). This situation is therefore more accurately described by Eq. (5). In order to understand the implications of Eq. (5) for the optical activity of such a system, it is instructive to consider the following simplified scenario.

Suppose we define a system that comprises a continuous closed surface $S$ that completely encloses a single point scatterer at a point $P_{1}$. Now consider two beams that follow different trajectories through this system but are both incident on the point scatterer from their different directions (see Fig. 3). For simplicity we assume that each beam is comprised of a uniform plane wave, and that beam 1 enters the closed surface $S$ at the point $A$ and exits at point $B$, while beam 2 enters at point $C$ and exits at point $D$. If both beams interact with the point scatterer en route through the system, some of the light from each beam will be scattered in all possible directions. We can therefore envisage a situation where light from both beams will be scattered to the same arbitrary point $Q$ on the surface $S$. If we then define a vector product $\mathbf{L}_{a b}=\mathbf{E}_{a} \times \mathbf{H}_{b}$, it can be shown that $\mathbf{L}_{12}=\mathbf{L}_{21}$ if the Poynting vectors $\mathbf{L}_{11}$ and $\mathbf{L}_{22}$ are parallel. As the two beams arriving at $Q$ from the scatterer $P_{1}$ will indeed be parallel, then the contribution of $\mathbf{L}_{12}-\mathbf{L}_{21}$ at $Q$ to the integral in Eq. (5) will be zero for all possible points for $Q$. This will be true

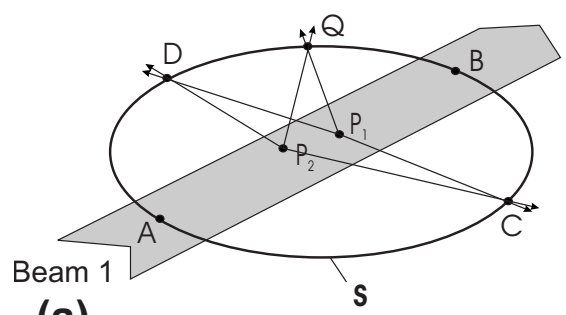

(a)

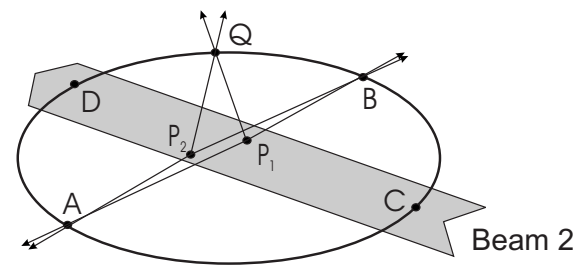

(b)

FIG. 3. The two field distributions used to evaluate reciprocity using Eq. (5) for a system $\mathbf{S}$ that encloses multiple point scatterers $\left(P_{1}, P_{2}\right.$, etc.) but no current sources. In (a) beam 1 enters $\mathbf{S}$ at $A$ and scatters off $P_{1}$ and $P_{2}$ before exiting at $B$. In (b) beam 2 enters $\mathbf{S}$ at $C$ and also scatters off $P_{1}$ and $P_{2}$ before exiting at $D$.

even if both of the scattered beams arriving at $Q$ are of a different amplitude, frequency and phase. Only if the two waves at the surface $S$ are incident on $S$ from different angles (or from different point scatterers $P_{1}$ and $P_{2}$ ) can their contributions to the integral in Eq. (5) be nonzero. For a system enclosing a single point scatterer this can only occur at point $A$ and point $C$. If there are multiple point scatterers within $S$ then Eq. (5) still holds because Eq. (2) and Eq. (3) both hold true. As any point scatterer will generate a field equivalent in form to that of a current element (or electric dipole) in Eq. (3), the fields on $S$ from any two point scatterers will also satisfy Eq. (5). Therefore, the only remaining fields for which Eq. (5) needs to be evaluated are those at the points on $S$ where the scattered field from one beam interacts with the input of the other beam. This only occurs at the points $A$ and $C$. At these points the two field contributions will be propagating in opposite directions and so $\mathbf{L}_{12}=-\mathbf{L}_{21}$.

Suppose that the linearly polarized input beam at $A$ (beam 1 ) has an electric field amplitude $E_{0}$ and a polarization azimuth $\phi_{1}$, while the output of beam 1 at $C$ has amplitude $E_{C}$ and a polarization azimuth $\chi_{1}=\phi_{1}+\Delta \phi_{1}$ (see Fig. 4). For the reverse propagation scenario (beam 2), let the input at $C$ also have amplitude $E_{0}$ but a polarization azimuth $\phi_{2}$, while its output at $A$ has amplitude $E_{A}$ and a polarization azimuth $\chi_{2}$ $=\phi_{2}+\Delta \phi_{2}$. It should be noted that in general $E_{C}$ and $\Delta \phi_{1}$ will be functions of the input polarization azimuth $\phi_{1}$, while $E_{A}$ and $\Delta \phi_{2}$ will be functions of $\phi_{2}$. Then, at $A$

$$
\left|\mathbf{L}_{12}\right|=-\left|\mathbf{L}_{21}\right|=E_{0} H_{A} \cos \left(\phi_{1}+\chi_{2}\right)
$$

while at $C$

$$
\left|\mathbf{L}_{12}\right|=-\left|\mathbf{L}_{21}\right|=-E_{0} H_{C} \cos \left(\phi_{2}+\chi_{1}\right) .
$$

From Eqs. (5), (9), and (10) it follows that 

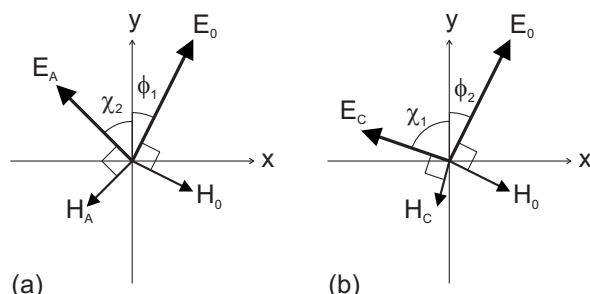

(b)

FIG. 4. (a) The electric and magnetic field vectors of the input beam (beam 1) and the scattered component of beam 2 as seen by an observer looking into the surface $S$ at point $A$. (b) The electric and magnetic field vectors of the input beam (beam 2) and the scattered component of beam 1 as seen by an observer looking into the surface $S$ at point $C$. These vector fields are then used to define the relevant terms in Eq. (11).

$$
\begin{aligned}
& H_{A} \cos \left(\phi_{1}+\phi_{2}+\Delta \phi_{2}\right) \cos \left(\beta_{1}\right) \\
& \quad=H_{C} \cos \left(\phi_{2}+\phi_{1}+\Delta \phi_{1}\right) \cos \left(\beta_{2}\right)
\end{aligned}
$$

where $\beta_{1}$ and $\beta_{2}$ are the angles of incidence of beam 1 and beam 2, respectively (as defined in Fig. 1). For the case of our PCM structures, if both beams are incident on the structure at normal incidence, but from opposite sides, then $\beta_{1}$ $=\beta_{2}=0$. The requirements of parity invariance demand that $H_{A}=H_{C}$, while the fourfold rotational symmetry of the structure means that $\Delta \phi_{1}$ must be independent of $\phi_{1}$ (and therefore the same for all values of $\phi_{1}$ ) and $\Delta \phi_{2}$ must equally be independent of $\phi_{2}$. Equation (11) can then only be satisfied for all possible inputs $\phi_{1}$ and $\phi_{2}$ if $\Delta \phi_{1}=\Delta \phi_{2}$. Therefore the polarization change induced by the system must be reciprocal. This will be true for almost any $2 \mathrm{D}$ structure at normal incidence with $C_{n}$ symmetry $(n \geq 3)$ or rotational invariance (such as 3D chiral crystals or solutions). The principal exception is that of the Faraday effect which is clearly nonreciprocal despite the system generally possessing an axis of rotational symmetry. This violation occurs because the condition $W=0$ in Eq. (8) is not satisfied. In the case of our PCM structures however, while Eq. (11) dictates that the zeroorder beam should be reciprocal at normal incidence, parity invariance, and the 2D chiral structure of our samples dictate that $\Delta \phi_{1}=-\Delta \phi_{2}$. These two requirements are clearly mutually antagonistic, and can only be satisfied if the zero-order beam experiences no polarization change due to the $2 \mathrm{D}$ chirality. Experiments have shown that this is indeed the case. However, Eq. (11) does not preclude nonreciprocal polarization rotations under more general circumstances.

If the symmetry requirements of the system are relaxed, then nonreciprocal behavior may be possible. For example, if the trajectory and polarization state of beam 2 are also the parity inverted form of beam 1 , then $\beta_{1}=-\beta_{2}, H_{A}=H_{C}, \phi_{1}$ $=-\phi_{2}$, and $\Delta \phi_{1}=-\Delta \phi_{2}$. As a result Eq. (11) is still satisfied. If such a scenario occurs when $\phi_{1}=\phi_{2}=0$ and the resulting polarization change is nonzero, then the polarization change will be nonreciprocal while still being compatible with the Lorentz reciprocity lemma.

It therefore appears that Lorentz reciprocity may permit nonreciprocal polarization changes (or gyrotropy) under certain circumstances. In order to test for these reciprocal ef-
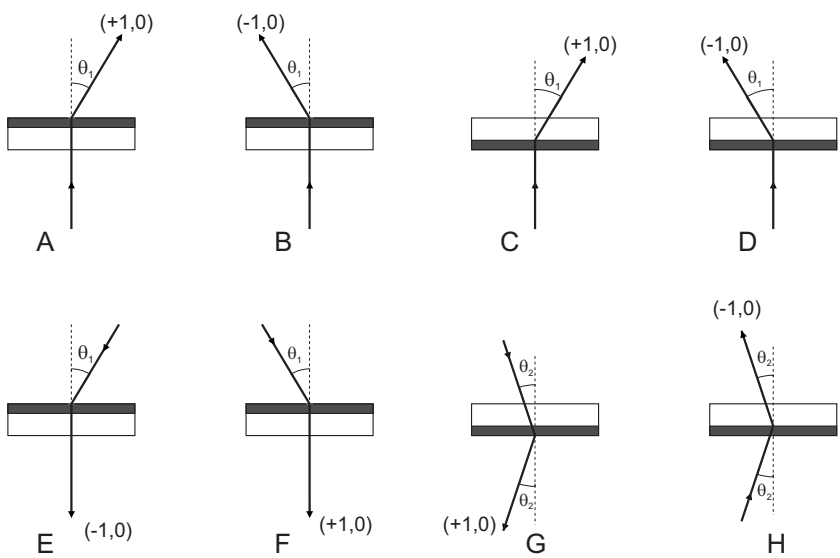

FIG. 5. An illustration of the eight different experimental configurations used to demonstrate nonreciprocity in the optical response of planar chiral materials. The appropriate angles of incidence and diffraction are indicated, as is the diffraction order in each case. For paths A, B, and $\mathrm{G}$ the beam is incident on the substrate side of the sample. For the other five configurations the incident beam strikes the $\mathrm{Si}_{3} \mathrm{~N}_{4}$ layer (shown in dark gray) first. The thicknesses of the substrate and the $\mathrm{Si}_{3} \mathrm{~N}_{4}$ layer are not shown to scale.

fects in the optical activity of our PCM samples, we have measured their gyrotropy for a number of different beam configurations illustrated in Fig. 5. These correspond to experimental arrangements where either the sample is reversed, the beam trajectory is reversed, or only the chirality of the sample is reversed. The results of our investigations are discussed in the next section.

\section{NONRECIPROCAL GYROTROPY}

In order to test the reciprocal properties of our planar chiral materials (PCMs) we have studied their gyrotropy for eight different experimental configurations (see Fig. 5). In the first configuration, the input beam is incident normal to the back face of the sample (i.e., on the unpatterned fused silica substrate) and the output polarization state of the $(+1,0)$ diffracted beam is measured in transmission for each of the different polarization states of the input beam. This configuration (which we will designate as path A) corresponds to a variation of the experimental setup illustrated in Fig. 1 where the angle of incidence $\beta=0^{\circ}$, and the angle of diffraction $\theta=+\theta_{1}$. The second configuration (path $\mathrm{B}$ ) we have considered is similar to path A with the only difference being that the opposite diffraction order $(-1,0)$ is chosen for the output beam. In this case the angle of diffraction $\theta=-\theta_{1}$. For the samples that we consider in this paper, $\theta_{1}$ is set by the ratio of the gammadion pitch $(\Lambda=5.0 \mu \mathrm{m})$ and the optical wavelength $(\lambda=632.8 \mathrm{~nm})$, and therefore $\theta_{1}=7.3^{\circ}$. These two configurations, path $\mathrm{A}$ and path $\mathrm{B}$, represent the two principal experimental arrangements used most frequently to investigate the optical activity of dielectric PCM structures in our previous papers $[8,12]$.

In Fig. 6 we have illustrated the typical polarization changes that are detected for both a left-handed PCM and its 

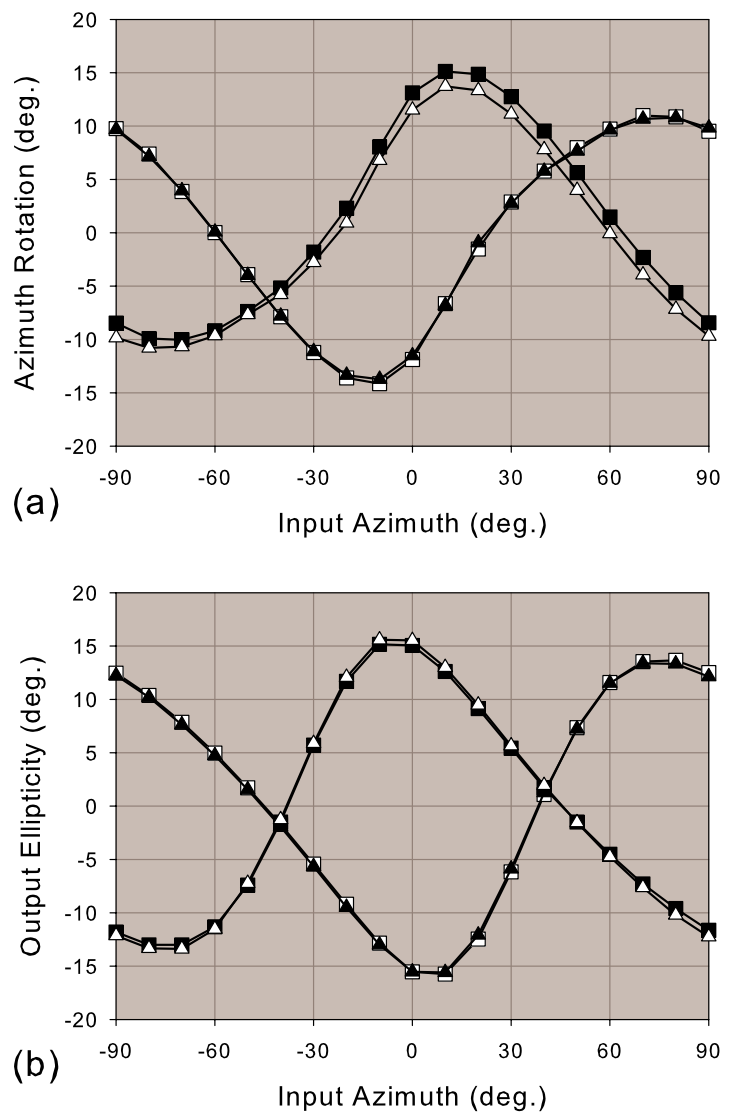

FIG. 6. (Color online) The azimuth rotation (a) and induced ellipticity (b) measured for the transmitted $(+1,0)$ and $(-1,0)$ beams diffracted from a left-handed PCM (sample W5BA42) and a right-handed PCM (sample W5BA45) when each is illuminated at normal incidence with linearly polarized light of wavelength $\lambda$ $=632.8 \mathrm{~nm}$. The experimental data shown correspond to that of path A $(\square)$ and path B $(\mathbf{\Delta})$ for sample W5BA42 (left-handed gammadion array) and path A (ם) for sample W5BA45 (right-handed gammadion array). Also shown is the predicted effect of parity inversion on the data for path B for sample W5BA42 $(\triangle)$. Each sample consists of an array of gammadion-shaped holes of pitch $\Lambda=5.0 \mu \mathrm{m}$ and gammadion arm-segment length $\xi=1.8 \mu \mathrm{m}$ etched into an 80-nm-thick film of $\mathrm{Si}_{3} \mathrm{~N}_{4}$ on a fused silica substrate. For sample W5BA42 (left-handed array) the bending angle $\alpha=-45^{\circ}$ while for sample W5BA45 (right-handed array) $\alpha=+45^{\circ}$.

right-handed enantiomer when each is illuminated with linearly polarized light at normal incidence using both path $\mathrm{A}$ and path $\mathrm{B}$ as the experimental configuration. It can be seen that the polarization changes for path $\mathrm{A}$ and path $\mathrm{B}$ for the left-handed PCM (sample W5BA42) are virtually identical, yet the equivalent polarization response for path A through the right-handed gammadion array (sample W5BA45) is very different. In each case the sample consisted of an array of gammadion-shaped holes of pitch $\Lambda=5.0 \mu \mathrm{m}$ and gammadion arm-segment length $\xi=1.8 \mu \mathrm{m}$ etched into an 80 -nm-thick film of $\mathrm{Si}_{3} \mathrm{~N}_{4}$ on a fused silica substrate. For the left-handed array (sample W5BA42) the bending angle $\alpha=-45^{\circ}$ while for sample W5BA45 (the right-handed array) $\alpha=+45^{\circ}$. The input beam in each case was linearly polarized with the input polarization state $\mathbf{J}_{0}=\mathbf{J}\left(\phi_{0}, \eta_{0}\right)$ being defined by the azimuthal angle $\left(\phi_{0}\right)$ only, the input ellipticity $\eta_{0}$ being zero. The measured output polarization state $\mathbf{J}_{1}$ $=\mathbf{J}\left(\phi_{1}, \eta_{1}\right)$ was generally elliptical with an azimuthal angle of $\phi_{1}$ and an ellipticity of $\eta_{1}$. The azimuth rotation in Fig. 6(a) is defined by $\Delta \phi=\phi_{1}-\phi_{0}$, while the ellipticity in Fig. 6(b) is $\eta_{1}$.

The two sets of results for path A and path B through sample W5BA42 illustrated in Fig. 6 are consistent with those reported previously for other structures [8]. We have already demonstrated how the gyrotropy exhibited by opposite diffraction orders from the same sample should be identical, provided that the sample surface has twofold rotational symmetry and the incident beam is aligned perpendicular to the sample surface [8]. The same effect is clearly evident in the gyrotropy of the $(+1,0)$ and $(-1,0)$ diffraction orders (path A and path B, respectively) for sample W5BA42 in Fig. 6 and is a consequence of the twofold rotational symmetry of the system. The data in Fig. 6 also shows how the gyrotropy effects for opposite enantiomers are related by parity. The application of parity inversion to path B through sample W5BA42 should yield an experimental configuration equivalent to path A through sample W5BA45. If the light-matter interaction for these dielectric structures is invariant under parity inversion, then the application of parity inversion to the data for path B through sample W5BA42 (as denoted by the black triangles in Fig. 6) should yield the negative polarization changes for both the ellipticity and azimuth for the negative input azimuth (see the white triangles in Fig. 6). By comparing the expected parity inverted data of path $\mathrm{B}$ through sample W5BA42 (white triangles) with the actual experimental data (black squares) for path A through sample W5BA45 (the parity inverted experimental arrangement), we see that there is no discernable difference in the two data sets. These results indicate that there are no significant manufacturing errors in our samples, and that the light-matter interaction for these dielectric structures is indeed invariant under parity inversion as has been shown previously [8]. We can also conclude that both samples must therefore possess almost perfect twofold rotational symmetry and must also be near perfect enantiomers of each other.

The data in Fig. 6 clearly demonstrate that the polarization changes exhibited by our dielectric PCMs are unquestionably a consequence of the chiral patterning of the sample surface as this is the only significant difference between the two samples. This is consistent with our previous work $[4,8]$ which has shown a strong correlation between the measured polarization change and the sense and magnitude of the surface chirality as quantified by a mathematical coefficient for the $2 \mathrm{D}$ chirality based on overlap integrals [1]. However, this does not prove that the polarization changes are necessarily the result of pure 2D chirality. They may also be the result of $3 \mathrm{D}$ chirality, or a complex mixture of the two types. A 3D chiral effect of this type arising from a combination of the $2 \mathrm{D}$ surface patterning and its spatial relationship to the substrate has been reported by Kuwata-Gonokami et al. [7] for metallic gammadion arrays. In this instance the gyrotropy was reciprocal, i.e., reversing the beam direction through the same sample had no effect on the resulting polarization change. As a consequence, there remained an element of doubt over whether the results we have reported previously 
for diffractive PCMs $[3,4,8,12]$ could also be due to $3 \mathrm{D}$ chiral effects rather than $2 \mathrm{D}$ chirality. In order to resolve this issue, we need to perform a number of experiments in which the input beam is incident on the opposite face of the sample from that employed for the data in Fig. 6.

Since the output beam for path A is not parallel to the incident beam, it is possible to define a reverse experimental configuration in one of two ways. Either the sample can be reversed by rotating it by $180^{\circ}$ about an axis perpendicular to the plane of incidence (path $\mathrm{C}$ ), or the beam trajectory can be reversed (path E). For path $\mathrm{C}$ the input beam is now incident normal $\left(\beta=0^{\circ}\right)$ to the front face of the sample (i.e., on the patterned $\mathrm{Si}_{3} \mathrm{~N}_{4}$ layer) and the polarization state of the $(+1,0)$ diffracted beam is again measured in transmission. For path E, the input beam is also incident on the front patterned face of the sample, but this time at an angle of incidence $\beta=-\theta_{1}$, and the $(-1,0)$ diffraction order now emerges from the substrate side of the sample in a direction normal to the sample surface. We can also reverse path B in a similar manner, either by reversing the sample orientation (path D), or by reversing the beam trajectory (path $\mathrm{F}$ ). For path $\mathrm{F}$ the angle of incidence $\beta=+\theta_{1}$, and it is the $(+1,0)$ diffraction order that now emerges normal to the sample surface on the substrate side of the sample. It is also worth noting that, if the sample possesses twofold rotational symmetry, path $\mathrm{C}$ and path $\mathrm{D}$ will generate identical polarization changes and can therefore be considered to be spatially equivalent, and path $\mathrm{E}$ and path $\mathrm{F}$ will also be equivalent and generate identical polarization changes, just as in path A and path B have previously been shown to be equivalent under spatial rotation [8].

In addition to the above configurations we can also define a beam trajectory for which a reversal of the sample orientation and a reversal of the beam propagation direction are identical. Such a situation will arise if the angles of incidence and diffraction are equal (i.e., $\beta=\theta= \pm \theta_{2}$ ), as illustrated by path $\mathrm{G}$ in Fig. 5. The reverse configuration is then defined by path $\mathrm{H}$. It should be appreciated that the equivalence of the two reversal processes in this case is also dependent on the sample surface topology exhibiting twofold rotational symmetry.

In order to investigate the possibility of non-reciprocal polarization properties for dielectric PCM enantiomers, we have measured the polarization change for a left-handed PCM for four of the eight configurations (A-H) illustrated in Fig. 5. In each case, the incident beam was linearly polarized with the input polarization state $\mathbf{J}_{0}=\mathbf{J}\left(\phi_{0}, \eta_{0}\right)$ being defined by the azimuthal angle $\left(\phi_{0}\right)$ only, the input ellipticity $\eta_{0}$ being zero. Each separate experiment involved measuring the polarization state of the output beam $\mathbf{J}_{1}=\mathbf{J}\left(\phi_{1}, \eta_{1}\right)$. The sample used for these experiments was almost identical to sample W5BA42 reported in Fig. 6, except that this time the thickness of the chirally patterned $\mathrm{Si}_{3} \mathrm{~N}_{4}$ film was $320 \mathrm{~nm}$. This sample (denoted by the name W3BA42) was a lefthanded gammadion array with a gammadion bending angle $\alpha=-45^{\circ}$. The results of these experiments are illustrated in Fig. 7.

The polarization change of a light beam of wavelength $632.8 \mathrm{~nm}$ diffracting through sample W3BA42 was measured for configurations $\mathrm{B}, \mathrm{D}, \mathrm{E}$, and $\mathrm{H}$. It can be seen that
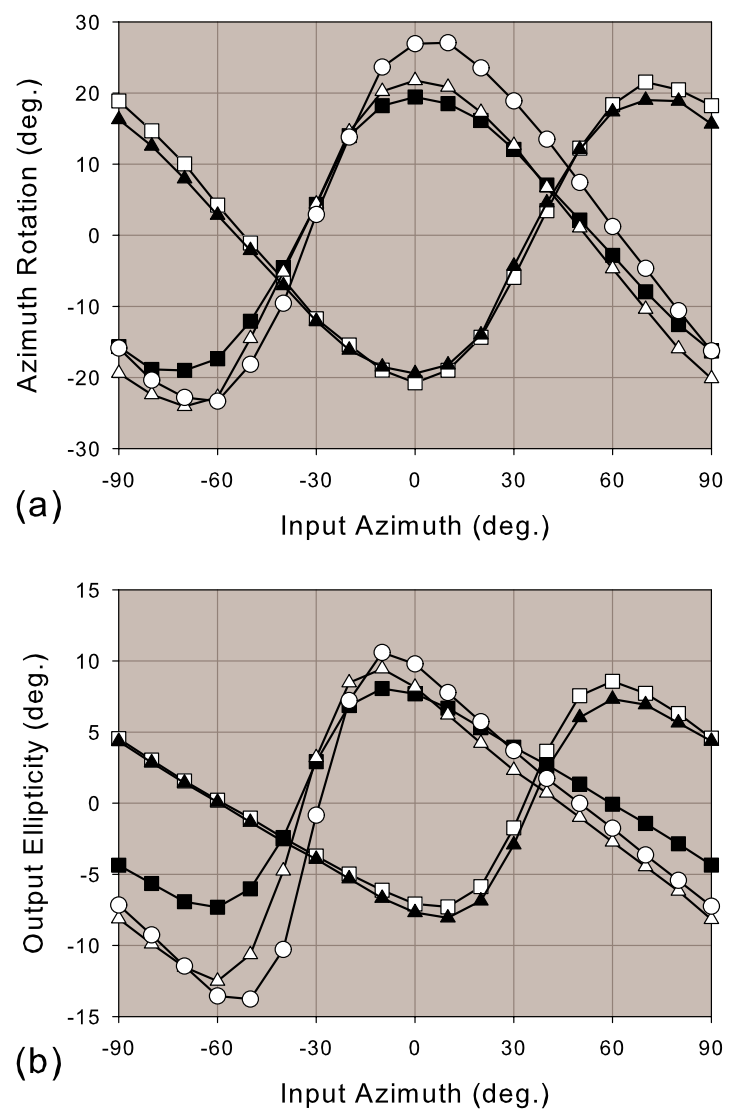

FIG. 7. (Color online) The polarization changes measured for light diffracted through a left-handed PCM (sample W3BA42) that is illuminated with linearly polarized light of wavelength $\lambda$ $=632.8 \mathrm{~nm}$ showing evidence of nonreciprocity. The experimental data shown correspond to that for path B $(\square)$, path D $(\mathbf{\square})$, path $\mathrm{E}$ $(\triangle)$, and path $H(\bigcirc)$ as defined in Fig. 5. Also shown is the predicted effect of parity inversion on the data for path D for sample W3BA42 (A). Sample W3BA42 consists of an array of gammadion-shaped holes of pitch $\Lambda=5.0 \mu \mathrm{m}$, gammadion armsegment length $\xi=1.8 \mu \mathrm{m}$, and gammadion bending angle $\alpha=$ $-45^{\circ}$ etched into an 320-nm-thick film of $\mathrm{Si}_{3} \mathrm{~N}_{4}$ on a fused silica substrate.

the results for path B (white squares) are very different from the results for path $\mathrm{D}$ (black squares). Thus reversing the sample direction dramatically alters the form of the polarization response to a form that is more indicative of that which one might expect to see for the opposite enantiomer. This suggests that the polarization changes may indeed be nonreciprocal, and the chirality that is responsible for these changes may be $2 \mathrm{D}$ in character. To verify this we have parity inverted the data for path D in Fig. 7. The resulting data set (represented by the black triangles) is almost identical to the data for path B (white squares). This demonstrates two facts. First, that the polarization response for path D through sample W3BA42 is almost identical to that which would be expected for both path A and path B through the opposite enantiomer (sample W3BA45). Thus the incident light diffracted along path D through the left-handed PCM (W3BA42) experiences a gyrotropic effect that is consistent with that of a right-handed PCM. Therefore, the chirality 
induced gyrotropy is indeed largely $2 \mathrm{D}$ in character. The second conclusion we can draw is that any $3 \mathrm{D}$ chiral effect is negligible, otherwise there would be a greater discrepancy between the path $\mathrm{B}$ data (white squares) and the parityinverted path D data (black triangles) in Fig. 7 than is actually observed. This suggests that the role of the substrate in influencing the gyrotropic effect is negligible, at least when the input beam is at normal incidence.

The results for path $\mathrm{B}$ and path $\mathrm{D}$ through sample W3BA42 are clearly suggestive of a form of nonreciprocal optical activity arising from the 2D chirality of the sample. It is a common misconception that such effects are forbidden in nonmagnetic systems such as the purely dielectric PCMs we are considering here due to the constraints of the Lorentz reciprocity lemma. However, we have already shown in the previous section that nonreciprocal optical activity does not necessarily violate the Lorentz reciprocity lemma [see Eq. (11)]. To verify that nonreciprocal optical activity is a possibility for these structures we have compared the polarization response of sample W3BA42 for path B in Fig. 5 with that for path $\mathrm{E}$. In this case, which more closely conforms to the geometry required for the testing of Lorentz reciprocity, path E represents the reverse beam trajectory of path A. However, as we have already shown in Fig. 6 and our earlier work [8], the polarization responses for paths $\mathrm{A}$ and $\mathrm{B}$ should always be equal for samples with twofold rotational symmetry. Therefore a comparison of the gyrotropy of path $\mathrm{E}$ with that for path $B$ should be equivalent to a comparison of path $E$ with path $\mathrm{A}$, and therefore represent a direct test of the nonreciprocal gyrotropy within a framework that is consistent with the Lorentz reciprocity lemma. These results are also shown in Fig. 7.

A comparison of the results shown in Fig. 7 for path B (white squares) and path $\mathrm{E}$ (white triangles) again indicates that the optical responses for the two paths differ significantly, and are therefore indicative of nonreciprocity. In fact the response for path $\mathrm{E}$ is similar to, but not identical to that from path D (black squares). This is perhaps not surprising given that the only difference between the two experimental configurations is a rather modest $\left(\theta_{1}=7.3^{\circ}\right)$ tilt in the sample orientation. A close examination of the data for paths $\mathrm{B}$ and $\mathrm{E}$ indicates that for no linearly polarized input state $\mathbf{J}_{0}$ $=\mathbf{J}\left(\phi_{0}, 0\right)$ is the polarization state of the output beam $\left(\mathbf{J}_{1}\right)$ the same for both paths B and E. Thus we can say that the polarization response is nonreciprocal. Moreover, if we compare the polarization changes at $\phi_{0}=0$ and $\phi_{0}= \pm 90^{\circ}$ where one would normally expect there to be no polarization change (these being the eigenstates of an achiral grating), we see that the polarization changes for paths $\mathrm{B}$ and $\mathrm{E}$ are almost exactly opposite, as predicted by Eq. (11). The small discrepancy can be accounted for by the small difference in the angles of incidence for the two paths.

Finally, if we consider the case of equal angles of incidence and diffraction (path $\mathrm{H}$ in Fig. 5) we see that the polarization response (as illustrated by the white circles in Fig. 7) is similar to that of path D (black squares) and path E (white triangles). This again is unsurprising given that the angle of tilt of the sample in this case $\left(\theta_{2}=3.6^{\circ}\right)$ is intermediate between that for path $\mathrm{D}\left(0^{\circ}\right)$ and that for path $\mathrm{E}\left(\theta_{1}\right.$ $=7 \cdot 3^{\circ}$ ).
It has been shown by other workers [7] that the polarization changes observed for zero-order beams transmitted through metallic films patterned with arrays of 2D chiral gammadions are reciprocal due to $3 \mathrm{D}$ chiral effects. This $3 \mathrm{D}$ chirality is conjectured to arise from the combination of the 2D surface chirality and the asymmetric material film arrangement along the direction normal to the patterned surface. However, we have seen that the polarization response of sample W3BA42 for path D (black squares) more closely resembles that predicted for the enantiomeric sample for path $\mathrm{B}$ despite the order in which the light beam propagates through the substrate and the $\mathrm{Si}_{3} \mathrm{~N}_{4}$ film being reversed. The two data sets for paths D and B then appear to be related by parity inversion, just as path A through W5BA42 and path B through W5BA45 are related by parity inversion. As the incident beam sees the 2D chirality of the patterned $\mathrm{Si}_{3} \mathrm{~N}_{4}$ film as having the same handedness for path D through W3BA42 as it would for both paths A and B through its enantiomer, this appears to confirm that it is the $2 \mathrm{D}$ chirality that is the dominant factor in defining the polarization change of these higher order diffracted beams, and not 3D chiral effects found by Kuwata-Gonokami et al. [7] for the zero-order case. While such a 3D chiral contribution may indeed be present in our samples, it is clearly dwarfed in magnitude by the more dominant $2 \mathrm{D}$ chiral contribution.

So far we have only shown that the polarization response of these dielectric PCM structures is nonreciprocal for linearly polarized input states. In the next section we will show that the polarization eigenstates of these PCM structures are also nonreciprocal. In order to demonstrate this, we will need to calculate the transfer (Jones) matrices for each path. These matrices relate the polarization state of the output beam $\left(\mathbf{J}_{1}\right)$ for a particular diffraction path or channel to the polarization state of the input beam $\mathbf{J}_{0}$, where $\mathbf{J}_{0}$ and $\mathbf{J}_{1}$ are the Jones vectors for each state. In the next section we will show how the transfer matrices can be calculated from the experimental data, and how the transfer matrix can be used to determine the eigenstates for a particular diffraction channel or path.

\section{POLARIZATION EIGENSTATES}

The polarization state of any monochromatic coherent beam can be characterized by two parameters: the polarization azimuth $\phi$ and the ellipticity $\eta$. It is also possible to describe the polarization state of any beam by the use of a Jones vector $\mathbf{J}$ such that

$$
\mathbf{J}(\phi, \eta)=\left[\begin{array}{l}
p \\
q
\end{array}\right]=p\left[\begin{array}{c}
1 \\
r e^{i \delta}
\end{array}\right]=p\left[\begin{array}{l}
1 \\
Z
\end{array}\right],
$$

where

$$
\begin{gathered}
p=\cos (\phi) \cos (\eta)+i \sin (\phi) \sin (\eta), \\
q=-\sin (\phi) \cos (\eta)+i \cos (\phi) \sin (\eta), \\
r^{2}=\frac{\tan ^{2}(\phi)+\tan ^{2}(\eta)}{1+\tan ^{2}(\phi) \tan ^{2}(\eta)}
\end{gathered}
$$

and 


$$
\tan (\delta)=\frac{\tan (\eta)\left[1+\tan ^{2}(\phi)\right]}{\tan (\phi)\left[\tan ^{2}(\eta)-1\right]}
$$

If $\mathbf{J}_{0}$ is the Jones vector representing the input state for a system, then the output state can be similarly described by a Jones vector $\mathbf{J}_{1}$. Each of the Jones vectors $\mathbf{J}_{0}$ and $\mathbf{J}_{1}$ can be characterized uniquely by an associated $Z$ parameter $\left(Z_{0}\right.$ and $Z_{1}$, respectively) as indicated in Eq. (12). If the optical system under consideration is also linear, then the input and output Jones vectors can be related by a $2 \times 2$ transfer matrix $\mathcal{A}$ such that

$$
\mu_{10} \mathbf{J}_{1}=\mathcal{A} \mathbf{J}_{0},
$$

where $\mu_{10}$ is a complex parameter representing the relative amplitudes and phases of the input and output states. The transfer matrix $\mathcal{A}$ can then be written in the form

$$
\mathcal{A}=\gamma\left[\begin{array}{cc}
1 & -Z_{c} Z_{b} \\
Z_{a} & Z_{c}
\end{array}\right]
$$

where the term $\gamma^{-2}=Z_{c}\left(1+Z_{a} Z_{b}\right)$. The three complex components of the transfer matrix $\mathcal{A}$ each represent the relative amplitudes and phases of the vertical $\left(E_{y}\right)$ and horizontal $\left(E_{x}\right)$ electric field components of the output wave $\mathbf{J}_{n}$ for different inputs. The term $Z_{a}$ represents the relative amplitude and phase of $E_{x}$ to $E_{y}$ when the input $\left(\mathbf{J}_{0}\right)$ is linearly polarized in the vertical direction. Likewise, $Z_{b}$ represents the relative amplitude and phase of $-E_{y}$ to $E_{x}$ when the input is linearly polarized in the horizontal direction. The third term $Z_{c}$ represents the relative amplitude and phase of $E_{y}$ in the first case to $E_{x}$ in the second.

For any linear optical system, the three complex components of its transfer matrix $\left(Z_{a}, Z_{b}\right.$, and $\left.Z_{c}\right)$ can be determined from polarization measurements of the type illustrated in Fig. 6 by measuring the polarization state of the output beam $\mathbf{J}_{1}$ $=\mathbf{J}\left(\phi_{1}, \eta_{1}\right)$ for three different linearly polarized input states. For example, $Z_{a}$ can be determined by measuring the output polarization state $\mathbf{J}_{1}$ for a vertically polarized linear input state (where $\phi_{0}=0^{\circ}$ and $\eta_{0}=0^{\circ}$ ). Then by substituting the values of $\Delta \phi=\phi_{1}-\phi_{0}$ and $\eta_{1}$ for $\phi$ and $\eta$, respectively, in both Eqs. (15) and (16) the terms $r_{a}$ and $\delta_{a}$ can be determined, where $Z_{a}=r_{a} e^{i \delta_{a}}$.

Similarly, the term $Z_{b}$ can be determined by measuring the output polarization state of the system for a horizontally polarized linear input state corresponding to $\phi_{0}=-90^{\circ}$ and $\eta_{0}$ $=0^{\circ}$, and again substituting the resulting $\Delta \phi=\phi_{1}-\phi_{0}$ and $\eta_{1}$ for $\phi$ and $\eta$, respectively, in Eqs. (15) and (16) to in order to determine the terms $r_{b}$ and $\delta_{b}$, and thence $Z_{b}$. The final term $Z_{c}$ can be determined by measuring the polarization change of the system for an input state that is a linear superposition of the inputs used to determine $Z_{a}$ and $Z_{b}$. If this superposition input state is linearly polarized at an azimuth $\phi_{0}=\phi_{s}$, and the resulting output polarization state of the system is $\mathbf{J}\left(\phi_{1}, \eta_{1}\right)$, then after determining $Z_{s}$ by substituting $\Delta \phi=\phi_{1}$ $-\phi_{0}$ and $\eta_{1}$ for $\phi$ and $\eta$, respectively, in Eqs. (15) and (16), the term $Z_{c}$ can be calculated using the equation

$$
Z_{c}=\frac{Z_{a}+Z_{a} Z_{s} \tan \phi_{s}+\tan \phi_{s}-Z_{s}}{Z_{s} \tan ^{2} \phi_{s}+Z_{b} Z_{s} \tan \phi_{s}+\tan \phi_{s}-Z_{b} \tan ^{2} \phi_{s}} .
$$

Once all the elements of the transfer matrix $\mathcal{A}$ have been determined, it is a relatively trivial task to determine the two polarization eigenstates of the system. These are the states for which the input and output polarization states for the diffraction channel are identical, and they can be represented by a pair of Jones vectors $\mathbf{J}_{+}$and $\mathbf{J}_{-}$, each with its characteristic $Z$ parameter, $Z_{+}$and $Z_{-}$such that $Z_{ \pm}=Z_{0}=Z_{1}$. Substituting $Z_{ \pm}$for $Z_{0}$ and $Z_{1}$ in Eq. (17) and eliminating the term $\mu_{10}$ yields the quadratic equation

$$
Z_{c} Z_{b} Z_{ \pm}^{2}+\left(Z_{c}-1\right) Z_{ \pm}+Z_{a}=0
$$

the solutions to which are

$$
Z_{ \pm}=r_{ \pm} e^{i \delta_{ \pm}}=\frac{1-Z_{c} \pm\left[\left(1-Z_{c}\right)^{2}-4 Z_{c} Z_{b} Z_{a}\right]^{1 / 2}}{2 Z_{c} Z_{b}} .
$$

From the parameters $r_{ \pm}$and $\delta_{ \pm}$the ellipticity $\left(\eta_{ \pm}\right)$and azimuthal angle $\left(\phi_{ \pm}\right)$of each polarization eigenstate can be determined using the relations

$$
\sin \left(2 \eta_{ \pm}\right)=\frac{2 r_{ \pm} \sin \left(\delta_{ \pm}\right)}{r_{ \pm}^{2}+1}
$$

and

$$
\tan \left(2 \phi_{ \pm}\right)=\frac{2 r_{ \pm} \cos \left(\delta_{ \pm}\right)}{r_{ \pm}^{2}-1} .
$$

We have calculated the two polarization eigenstates $\mathbf{J}_{+}$and

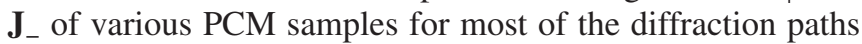
outlined in Fig. 5. These eigenstates are listed in Table I in terms of the ellipticity and azimuthal angle in each case. In contrast to the case of a conventional 3D chiral system, it can be seen that none of these eigenstates is circularly polarized. In fact, a purely $2 \mathrm{D}$ chiral system with fourfold $\left(C_{4}\right)$ rotational symmetry would also be expected to have circular eigenstates, but unlike 3D chiral systems, the eigenvalues should interchange for opposite propagation directions. Instead, all the eigenstates listed in Table I appear to be almost linear but with small components of ellipticity. The amount of ellipticity appears to be dependent (at least in part) on the $\mathrm{Si}_{3} \mathrm{~N}_{4}$ film thickness and is greatest for the PCMs with 80nm-thick $\mathrm{Si}_{3} \mathrm{~N}_{4}$ films and smallest for those with 160-nmthick films. This behavior does however appear to correlate with another finding that we reported previously [8], whereby $2 \mathrm{D}$ chiral features etched into quartz substrates without the $\mathrm{Si}_{3} \mathrm{~N}_{4}$ film failed to exhibit any chirality dependent polarization changes at all. This suggests that the $\mathrm{Si}_{3} \mathrm{~N}_{4}$ film plays an important role in generating the polarization changes, possibly by acting as a Fabry-Perot resonant cavity that induces different phase retardations on the vertical $(s$-polarized) and horizontal ( $p$-polarized) linear components of the elliptical wave as it traverses the cavity. This would then also explain how changing the $\mathrm{Si}_{3} \mathrm{~N}_{4}$ film thickness affects the ellipticity of the polarization eigenstates, but not the azimuthal angle. The azimuthal angle is relatively insensitive 
TABLE I. Polarization eigenstates $\mathbf{J}_{+}=\mathbf{J}\left(\phi_{+}, \eta_{+}\right)$and $\mathbf{J}_{-}=\mathbf{J}\left(\phi_{-}, \eta_{-}\right)$for the diffraction channels A, B, D, E, and H (as defined in Fig. 5) transmitted through left-handed (L) and right-handed (R) arrays of gammadion-shaped holes in $\mathrm{Si}_{3} \mathrm{~N}_{4}$ films of thickness $t$. Each diffraction path is additionally characterized by its angle of incidence $(\beta)$, the diffraction angle $(\theta)$, the diffraction order and the direction of propagation (substrate first or $\mathrm{Si}_{3} \mathrm{~N}_{4}$ layer first). The chirality of the gammadion array ( $\mathrm{L}$ or $\mathrm{R}$ ) is determined by the gammadion bending angle $(\alpha)$. For each sample the gammadion arm-segment length $\xi=1.8 \mu \mathrm{m}$ and the pitch $\Lambda=5.0 \mu \mathrm{m}$. The term $\Delta \Theta$ represents the degree of nonorthogonality between the two eigenstates.

\begin{tabular}{|c|c|c|c|c|c|c|c|c|c|c|c|c|c|c|}
\hline Set & Sample & $t / \mathrm{nm}$ & $\mathrm{L} / \mathrm{R}$ & $\alpha$ & Path & Direction & Order & $\beta$ & $\theta$ & $\phi_{+}$ & $\eta_{+}$ & $\phi_{-}$ & $\eta_{-}$ & $\Delta \Theta$ \\
\hline (a) & W5BA42 & 80 & $\mathrm{~L}$ & $-45^{\circ}$ & A & Substrate & $(+1,0)$ & $0^{\circ}$ & $+7.3^{\circ}$ & $+35.6^{\circ}$ & $+6.49^{\circ}$ & $-53.7^{\circ}$ & $+7.34^{\circ}$ & $27.7^{\circ}$ \\
\hline (b) & W5BA42 & 80 & $\mathrm{~L}$ & $-45^{\circ}$ & B & Substrate & $(-1,0)$ & $0^{\circ}$ & $-7.3^{\circ}$ & $+35.4^{\circ}$ & $+6.60^{\circ}$ & $-54.0^{\circ}$ & $+7.05^{\circ}$ & $27.3^{\circ}$ \\
\hline (c) & W5BA45 & 80 & $\mathrm{R}$ & $+45^{\circ}$ & A & Substrate & $(+1,0)$ & $0^{\circ}$ & $+7.3^{\circ}$ & $-35.4^{\circ}$ & $-5.59^{\circ}$ & $+56.6^{\circ}$ & $-9.11^{\circ}$ & $29.7^{\circ}$ \\
\hline (d) & W5BA45 & 80 & $\mathrm{R}$ & $+45^{\circ}$ & B & Substrate & $(-1,0)$ & $0^{\circ}$ & $-7.3^{\circ}$ & $-35.3^{\circ}$ & $-5.15^{\circ}$ & $+56.5^{\circ}$ & $-8.69^{\circ}$ & $27.9^{\circ}$ \\
\hline (e) & W2BA42 & 160 & $\mathrm{~L}$ & $-45^{\circ}$ & A & Substrate & $(+1,0)$ & $0^{\circ}$ & $+7.3^{\circ}$ & $+37.9^{\circ}$ & $-0.42^{\circ}$ & $-53.7^{\circ}$ & $-0.69^{\circ}$ & $3.88^{\circ}$ \\
\hline (f) & W2BA45 & 160 & $\mathrm{R}$ & $+45^{\circ}$ & A & Substrate & $(+1,0)$ & $0^{\circ}$ & $+7.3^{\circ}$ & $-37.6^{\circ}$ & $+0.33^{\circ}$ & $+54.9^{\circ}$ & $-0.70^{\circ}$ & $5.00^{\circ}$ \\
\hline (g) & W6BA42 & 240 & $\mathrm{~L}$ & $-45^{\circ}$ & A & Substrate & $(+1,0)$ & $0^{\circ}$ & $+7.3^{\circ}$ & $+32.8^{\circ}$ & $+3.40^{\circ}$ & $-54.3^{\circ}$ & $-0.38^{\circ}$ & $8.43^{\circ}$ \\
\hline (h) & W6BA45 & 240 & $\mathrm{R}$ & $+45^{\circ}$ & A & Substrate & $(+1,0)$ & $0^{\circ}$ & $+7.3^{\circ}$ & $-29.0^{\circ}$ & $-2.11^{\circ}$ & $+56.2^{\circ}$ & $+0.08^{\circ}$ & $10.4^{\circ}$ \\
\hline (i) & W3BA42 & 320 & $\mathrm{~L}$ & $-45^{\circ}$ & B & Substrate & $(-1,0)$ & $0^{\circ}$ & $-7.3^{\circ}$ & $+36.6^{\circ}$ & $-1.55^{\circ}$ & $-51.9^{\circ}$ & $-1.69^{\circ}$ & $7.13^{\circ}$ \\
\hline (j) & W3BA42 & 320 & $\mathrm{~L}$ & $-45^{\circ}$ & $\mathrm{D}$ & $\mathrm{Si}_{3} \mathrm{~N}_{4}$ & $(-1,0)$ & $0^{\circ}$ & $-7.3^{\circ}$ & $-34.9^{\circ}$ & $-0.16^{\circ}$ & $+55.8^{\circ}$ & $+0.71^{\circ}$ & $1.87^{\circ}$ \\
\hline (k) & W3BA42 & 320 & $\mathrm{~L}$ & $-45^{\circ}$ & $\mathrm{E}$ & $\mathrm{Si}_{3} \mathrm{~N}_{4}$ & $(-1,0)$ & $-7.3^{\circ}$ & $0^{\circ}$ & $-34.6^{\circ}$ & $+0.45^{\circ}$ & $+51.9^{\circ}$ & $-2.50^{\circ}$ & $8.17^{\circ}$ \\
\hline (1) & W3BA42 & 320 & $\mathrm{~L}$ & $-45^{\circ}$ & $\mathrm{H}$ & $\mathrm{Si}_{3} \mathrm{~N}_{4}$ & $(-1,0)$ & $-3.6^{\circ}$ & $-3.6^{\circ}$ & $-30.4^{\circ}$ & $+1.71^{\circ}$ & $+61.7^{\circ}$ & $-3.36^{\circ}$ & $5.34^{\circ}$ \\
\hline
\end{tabular}

to the $\mathrm{Si}_{3} \mathrm{~N}_{4}$ film thickness, but does appear to be strongly influenced by the $2 \mathrm{D}$ chirality of the surface patterning.

It is evident that for all the samples studied, the azimuthal angle of each eigenstate in Table I is strongly rotated from the $s$-polarized $\left(\phi=0^{\circ}\right)$ and $p$-polarized $\left(\phi=90^{\circ}\right)$ linear states that represent the eigenstates across a uniform heterogeneous dielectric interface at a nonzero angle of incidence $\left(\beta \neq 0^{\circ}\right)$. In addition, the two eigenstates for each path are generally nonorthogonal with the degree of nonorthogonality being strongly dependent on the ellipticity of the two eigenstates. We have quantified this nonorthogonality using the parameter $\Delta \Theta=180^{\circ}-\Theta$, where $\cos (\Theta)=\mathbf{S}_{+} \cdot \mathbf{S}_{-}$and the terms $\mathbf{S}_{+}$and $\mathbf{S}_{-}$are three-dimensional Stokes vectors for the two respective eigenstates $\mathbf{J}_{+}$and $\mathbf{J}_{-}$. Each Stokes vector $\mathbf{S}(\phi, \eta)$ has three mutually orthogonal components $S_{1}$ $=\cos (2 \eta) \cos (2 \phi), S_{2}=\cos (2 \eta) \sin (2 \phi)$, and $S_{3}=\sin (2 \eta)$. The term $\Theta$ therefore represents the angle between the two Stokes vectors $\mathbf{S}_{+}$and $\mathbf{S}_{-}$in the Stokes parameter space. Thus, the two eigenstates are defined to be orthogonal if their respective Stokes vectors are antiparallel $\left(\Theta=180^{\circ}\right)$. The data in Table I indicates that while $\Delta \Theta$ is seen to vary for different PCM samples and for different optical diffraction paths, in no instance can we claim with any certainty that the eigenstates for a particular path through a given sample are orthogonal, and in general it appears that for most cases nonorthogonality is the norm.

It can be seen by comparing data set (a) with data set (b) in Table I, and data set (c) with data set (d), that the eigenstates for opposite diffraction orders from the same sample are virtually identical when the angle of incidence is zero (i.e., $\beta=0^{\circ}$ ). We have previously shown that the optical activity of opposite diffraction orders should be identical if the sample and experimental arrangement possess twofold rotational symmetry [8]. They should therefore also share the same transfer matrix and eigenstates. The data in Table I suggests that this is indeed the case.
In addition, we can see from data sets (a)-(h) that the eigenstates for a given diffraction path through one enantiomer are also related by parity to the eigenstates of the same path through the opposite enantiomer. This is further evidence to support the invariance of the light-matter interaction in these structures under parity inversion. However, it is also true that the eigenstates for similar beam trajectories but opposite sample orientations for the same enantiomer also appear to be related by parity. The eigenstates for path B through sample W3BA42 [see data set (i)] are almost the mirror image of those for path $\mathrm{D}$ through the same sample [see data set $(j)]$. This suggests that the transfer matrices for these two paths are also related by parity inversion, and is further evidence that the polarization changes are the consequence of $2 \mathrm{D}$ chirality. It can also be seen that the eigenstates for path $\mathrm{E}$ are also very similar to those for path $\mathrm{D}$ as would be expected from inspection of the polarization data in Fig. 7. Thus reversing the propagation direction along the diffraction channel (such as from path A to path E) also appears to almost completely reverse the eigenstates.

It has been predicted recently by Prosvirnin and Zheludev [14] using numerical simulation techniques that the eigenstates for opposite directions of propagation along the same optical path through a metallic PCM structure formed from arrays of gammadions or rosettes should be biorthogonal. It should be noted that their definition of biorthogonality relates to one eigenstate for the forward path (path B) being orthogonal to one of the eigenstates of the reverse path (path $\mathrm{E})$, but crucially also assumes that the azimuthal angle is measured from the same viewpoint for both paths (while curiously the ellipticity is not). This is different from our definition where both the ellipticity and the azimuthal angle are always measured from the point of view of an observer looking into the on-coming beam (see Fig. 1). Taking into account this change of basis, the eigenstates listed in Table I do suggest that a biorthogonal relationship between these 
eigenstates, as defined by Prosvirnin and Zheludev, may also exist for our dielectric PCMs. However, the relatively small ellipticity seen for most of the eigenstates for our samples coupled with the magnitude of our experimental error, means that we cannot be definitive in our verification of this relationship.

So far we have shown that light diffracted through dielectric PCM samples along a nonzero order diffraction channel (such as path B) exhibits changes to its polarization state that are nonreciprocal for all linearly polarized input states if either the sample orientation is reversed (path $\mathrm{D}$ ) or the direction of propagation is reversed (path E). Now we have also shown that the eigenstates for both reversal processes are also nonreciprocal. It may be tempting to think that it can therefore be assumed that the polarization changes for these PCM structures are nonreciprocal for all input polarization states. However, this is not the case. Just as it is possible to find two eigenstates for any diffraction path, so too is it possible to find two input states for which the polarization change is completely reciprocal.

Suppose that the transfer matrix for a given path $i$ through a particular sample is given by

$$
\mathcal{A}_{i}=\gamma_{i}\left[\begin{array}{cc}
1 & -Z_{c i} Z_{b i} \\
Z_{a i} & Z_{c i}
\end{array}\right] \text {. }
$$

It can then be shown that two paths $m$ and $n$ will be reciprocal for an input state characterized by a $Z$ factor $Z_{0}$ in its Jones vector [see Eq. (12)] if $Z_{0}$ is a solution to the equation

$$
\begin{aligned}
& {\left[Z_{c m}\left(1+Z_{b m} Z_{a n}\right)-Z_{c n}\left(1+Z_{b n} Z_{a m}\right)\right] Z_{0}+Z_{c m} Z_{c n}\left(Z_{b m}-Z_{b n}\right) Z_{0}^{2}} \\
& \quad+Z_{a m}-Z_{a n}=0 .
\end{aligned}
$$

Moreover, if the two paths $m$ and $n$ are related by parity inversion then $Z_{a m}=-Z_{a n}, Z_{b m}=-Z_{b n}$, and $Z_{c m}=+Z_{c n}$. The solutions to Eq. (25) are then

$$
Z_{1}^{2}=Z_{c}^{2} Z_{0}^{2}=-\frac{Z_{a}}{Z_{b}}
$$

where $Z_{1}$ is the $Z$ factor of the output state $\mathbf{J}_{1}=\mathbf{J}\left(\phi_{1}, \eta_{1}\right)$ in each case.

We have calculated these reciprocal input and output states for two scenarios. In the first of these the forward and reverse paths are defined by paths $\mathrm{B}$ and $\mathrm{E}$, respectively. In the second they are defined by paths $\mathrm{G}$ and $\mathrm{H}$. The input and output states for each scenario are listed in Table II. In both cases the input states required for reciprocity are almost linear and close to (but not identical to) the eigenstates in each case. In addition it can be seen that the two reciprocal states for paths $\mathrm{G}$ and $\mathrm{H}$ are related by parity inversion, just as the paths themselves are related by parity.

It should be noted that for the second reciprocal state scenario in Table II we have deduced the transfer matrix for path $\mathrm{G}\left(\mathcal{A}_{\mathrm{G}}\right)$ by first deriving the transfer matrix for path $\mathrm{H}$ $\left(\mathcal{A}_{\mathrm{H}}\right)$ using the polarization data in Fig. 7 (represented by the white circles) and applying parity inversion to it. The validity of such a procedure is supported by the level of agreement of the parity inverted path D data for sample W3BA42 in Fig. 7 (as represented by the black triangles) and the path B data (white squares). If we define a parity operator $\mathcal{P}$ such that
TABLE II. A summary of the two reciprocal input polarization states $\left[\mathbf{J}_{0}=\mathbf{J}\left(\phi_{0}, \eta_{0}\right)\right]$ for sample $\mathrm{W} 3 \mathrm{BA} 42$ in the cases where (i) the input path is path $\mathrm{B}$ and the reverse path is path $\mathrm{E}$ (or vice versa) and (ii) when the input path is path $\mathrm{H}$ and the reverse path is path $\mathrm{G}$ (or vice versa), and their associated output states $\left[\mathbf{J}_{1}\right.$ $\left.=\mathbf{J}\left(\phi_{1}, \eta_{1}\right)\right]$ in each case.

\begin{tabular}{cccccc}
\hline \hline State & Path & $\phi_{0}$ & $\eta_{0}$ & $\phi_{1}$ & $\eta_{1}$ \\
\hline 1 & $\mathrm{~B}, \mathrm{E}$ & $-39.8^{\circ}$ & $+1.44^{\circ}$ & $-46.0^{\circ}$ & $-1.78^{\circ}$ \\
2 & $\mathrm{~B}, \mathrm{E}$ & $+41.6^{\circ}$ & $-2.63^{\circ}$ & $+47.4^{\circ}$ & $-0.84^{\circ}$ \\
1 & $\mathrm{G}, \mathrm{H}$ & $-39.5^{\circ}$ & $+5.36^{\circ}$ & $-52.7^{\circ}$ & $+0.51^{\circ}$ \\
2 & $\mathrm{G}, \mathrm{H}$ & $+39.5^{\circ}$ & $-5.36^{\circ}$ & $+52.7^{\circ}$ & $-0.51^{\circ}$ \\
\hline \hline
\end{tabular}

$$
\mathcal{P}=\left[\begin{array}{cc}
1 & 0 \\
0 & -1
\end{array}\right]
$$

then $\mathcal{A}_{\mathrm{G}}$ will be related to $\mathcal{A}_{\mathrm{H}}$ by

$$
\mathcal{A}_{\mathrm{G}}=\mathcal{P} \mathcal{A}_{\mathrm{H}} \mathcal{P}^{-1}=\mathcal{P} \mathcal{A}_{\mathrm{H}} \mathcal{P} \text {. }
$$

As a result, the transfer matrix for path $\mathrm{G}$ will be

$$
\mathcal{A}_{\mathrm{G}}=\gamma_{\mathrm{H}}\left[\begin{array}{cc}
1 & Z_{c \mathrm{H}} Z_{b \mathrm{H}} \\
-Z_{a \mathrm{H}} & Z_{c \mathrm{H}}
\end{array}\right] \text {. }
$$

It can be seen from Eq. (29) that the matrix $\mathcal{A}_{\mathrm{G}}$ is similar in form to the inverse of matrix $\mathcal{A}_{\mathrm{H}}$ given by

$$
\mathcal{A}_{\mathrm{H}}^{-1}=\gamma_{\mathrm{H}}\left[\begin{array}{rr}
Z_{c \mathrm{H}} & +Z_{c \mathrm{H}} Z_{b \mathrm{H}} \\
-Z_{a \mathrm{H}} & 1
\end{array}\right] .
$$

If $Z_{c \mathrm{H}}=1$, then $\mathcal{A}_{\mathrm{G}}=\mathcal{A}_{\mathrm{H}}^{-1}$. This suggests that, under certain circumstances, the polarization changes due to a $2 \mathrm{D}$ chiral structure could be reversible.

\section{NONRECIPROCAL INTENSITY MODULATIONS}

In the previous two sections we demonstrated that the polarization response of a dielectric planar chiral material (PCM) is nonreciprocal for all linearly polarized input states, and also for all elliptical input states except for two unique states defined by Eq. (25) and presented in Table II. In this section we will also show that nonreciprocity is also manifested in the intensity of the various diffracted beams.

It was shown in the previous section that the transfer matrix for any given diffraction channel can be defined in a form given by Eq. (18). It can be seen that the general form of this transfer matrix is nonunitary. One consequence of this is that the amplitude, phase, and intensity of the output beam should all depend on the polarization state of the input beam $\mathbf{J}=\mathbf{J}\left(\phi_{0}, \eta_{0}\right)$. For the case of the output intensity $\left(I_{1}\right)$, this functional dependence can be shown to be of the form

$$
\begin{aligned}
I_{1}= & I_{0} \beta_{0} \gamma^{*} \gamma\left[\alpha_{0}+\alpha_{1} \cos \left(2 \eta_{0}\right) \cos \left(2 \phi_{0}\right)\right. \\
& \left.+\alpha_{2} \cos \left(2 \eta_{0}\right) \sin \left(2 \phi_{0}\right)+\alpha_{3} \sin \left(2 \eta_{0}\right)\right],
\end{aligned}
$$

where the parameters $\alpha_{0}-\alpha_{3}$ are those given by

$$
2 \alpha_{0}=1+x_{a}^{2}+y_{a}^{2}+\left(x_{c}^{2}+y_{c}^{2}\right)\left(1+x_{b}^{2}+y_{b}^{2}\right),
$$




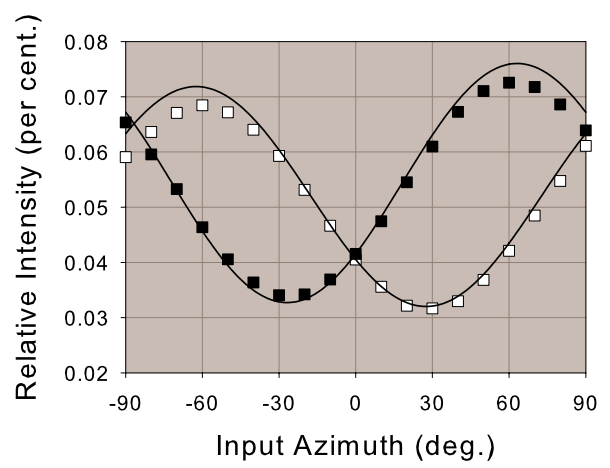

FIG. 8. (Color online) The observed variation in relative intensity for the $(-1,0)$ beam diffracted along path $\mathrm{B}$ through a lefthanded PCM (sample W5BA42, $\square$ ) and the $(+1,0)$ beam diffracted along path A through a right-handed PCM (sample W5BA45, when each is illuminated at normal incidence with linearly polarized light of wavelength $\lambda=632.8 \mathrm{~nm}$. Each sample consists of an array of gammadion-shaped holes of pitch $\Lambda=5.0 \mu \mathrm{m}$ and gammadion arm-segment length $\xi=1.8 \mu \mathrm{m}$ etched into an 80-nm-thick film of $\mathrm{Si}_{3} \mathrm{~N}_{4}$ on a fused silica substrate. For sample W5BA42 (lefthanded array) the bending angle $\alpha=-45^{\circ}$ while for sample W5BA45 (right-handed array) $\alpha=+45^{\circ}$. The solid curves represent the predicted intensity in each case based on the respective transfer matrices derived using the data in Fig. 6.

$$
\begin{gathered}
2 \alpha_{1}=1+x_{a}^{2}+y_{a}^{2}-\left(x_{c}^{2}+y_{c}^{2}\right)\left(1+x_{b}^{2}+y_{b}^{2}\right), \\
\alpha_{2}=x_{c}\left(x_{b}-x_{a}\right)-y_{c}\left(y_{a}+y_{b}\right),
\end{gathered}
$$

and

$$
\alpha_{3}=y_{c}\left(x_{b}-x_{a}\right)+x_{c}\left(y_{a}+y_{b}\right)
$$

while the term $\beta_{0}$ is a constant that represents a measure of the diffraction efficiency for this particular diffraction channel. The terms $x_{a}, x_{b}$, and $x_{c}$ are the real parts of the terms $Z_{a}$, $Z_{b}$, and $Z_{c}$ in the transfer matrix $\mathcal{A}$ defined in Eq. (18), while $y_{a}, y_{b}$, and $y_{c}$ are the respective imaginary parts. $I_{0}$ is the intensity of the input beam.

We have measured the output intensity of a number of PCM samples and their enantiomers for an experimental configuration corresponding to path B in Fig. 5 and over a range of linearly polarized input states $\mathbf{J}_{0}=\mathbf{J}\left(\phi_{0}, 0\right)$. In most cases the relative intensity $\left(I_{1} / I_{0}\right)$ of the $(-1,0)$ diffracted beam was found to exhibit a strong modulation as a function of $\phi_{0}$ (see Fig. 8). The amplitude of this modulation effect was found to vary significantly from sample to sample depending upon the thickness of the $\mathrm{Si}_{3} \mathrm{~N}_{4}$ film, but was also found to agree well with the predictions of the transfer matrix (solid curves in Fig. 8). In each case in Fig. 8 the only fitting parameter used to scale the predicted data (solid curves) to the experimental data (black and white squares) was the term $\beta_{0}$ in Eq. (31).

The data in Fig. 8 illustrates how the intensity modulation is different for opposite enantiomers. Only at linearly polarized input states corresponding to $\phi_{0}=0^{\circ}$ and $\phi_{0}= \pm 90^{\circ}$ are the output intensities the same for opposite enantiomers. This should also be true for paths $\mathrm{G}$ and $\mathrm{H}$ through the same samples.
Under parity inversion the terms $\alpha_{0}$ and $\alpha_{1}$ in Eq. (31) remain unchanged while $\alpha_{2}$ and $\alpha_{3}$ change sign. Thus for a pair of opposite beam trajectories that are related by parity inversion such as paths $\mathrm{G}$ and $\mathrm{H}$, the output intensity will be reciprocal, not only for the linear states $\phi_{0}=0^{\circ}$ and $\phi_{0}$ $= \pm 90^{\circ}$, but also for all input states $\mathbf{J}_{0}=\mathbf{J}\left(\phi_{0}, \eta_{0}\right)$ for which the condition $\sin \left(2 \phi_{0}\right)=k_{0} \tan \left(2 \eta_{0}\right)$ is satisfied with $k_{0}=$ $-\alpha_{3} / \alpha_{2}$. For path $\mathrm{H}$ through sample W3BA42 we found that $k_{0}=0.0687$ while the data in Table II indicate that the ratio $\sin \left(2 \phi_{0}\right) / \tan \left(2 \eta_{0}\right)=-5.18$ for both reciprocal states. Thus the criterion required for reciprocity of the polarization change as set out in Eq. (26) is different from that required for the transmitted intensity. Therefore, overall we can say that the optical properties of nonzero order diffracted beams are nonreciprocal for all input states and conditions as, in general, our PCM samples will not be reciprocal for both changes in polarization and changes in their transmitted intensity simultaneously.

\section{SUMMARY}

Our aim in undertaking this work has been to investigate possible manifestations of nonreciprocity in the optical activity of dielectric planar chiral materials (PCMs) arising from the presence of 2D chirality in these structures. The PCM samples that we have studied all consisted of $\mathrm{Si}_{3} \mathrm{~N}_{4}$ films patterned with regular square arrays of asymmetric elements that were supported on transparent fused silica substrates. As a result, the transmitted light formed a well-defined square diffraction pattern due to the array pitch of these structures being significantly greater than the optical wavelength used for their characterization $(\lambda=632.8 \mathrm{~nm})$.

We have shown that when these structures are illuminated with linearly polarized light, most of the transmitted diffracted beams exhibit significant rotations in their polarization azimuth compared to the input beam, and also incorporate substantial amounts of ellipticity. We have shown that the sense of these polarization changes for each PCM sample is reversed if the direction of propagation of the beam through the sample is reversed. As 2D chiral structures are also seen to reverse their sense of chirality when viewed from the opposite direction, this is incontrovertible evidence that the polarization changes are directly correlated with the sense of 2D (or planar) chirality of the structure as seen by the incident beam. They therefore arise as a direct consequence of the $2 \mathrm{D}$ chirality and not from $3 \mathrm{D}$ chirality as has been reported previously by other workers for the case of the zero-order beam transmitted through metallic PCMs [7]. These two results are not necessarily contradictory as Kuwata-Gonokami et al. [7] did not investigate the properties of higher order diffracted beams, while the zero-order beams transmitted by our dielectric PCMs exhibited no discernable optical activity.

In this paper we have rigorously demonstrated that the gyrotropy produced by the 2D chirality in our PCMs is completely nonreciprocal in character. Despite this, it does not violate the Lorentz reciprocity lemma. For a $2 \mathrm{D}$ chiral structure with $C_{4}$ symmetry, only the zero-order beam at normal incidence is prevented by the Lorentz reciprocity condition 
from exhibiting any optical activity arising solely from the $2 \mathrm{D}$ chirality, and consequently any polarization changes seen in the zero order beam must be due to residual 3D chiral effects as demonstrated previously by Kuwata-Gonokami et al. [7]. In our diffractive structures the diffraction process permits nonreciprocal 2D chiral effects by breaking the $C_{4}$ symmetry of the structure. This in turn results in a gyrotropy that is a strong oscillatory function of the input polarization azimuth, rather than the more usual rotationally invariant form seen for conventional 3D chiral structures. It also results in large intensity modulations of the transmitted beams that are strong functions of the input polarization state.

The work presented herein represents the first comprehensive analysis of reciprocity, its causes and its limitations, in asymmetric diffractive materials. The nonreciprocal effects that we have identified also have implications for the reversibility of the light-matter interaction in these structures. Such effects will be considered in a subsequent publication.

\section{ACKNOWLEDGMENTS}

The authors would like to thank the staff of Innos, Ltd., for their help during the fabrication of the planar chiral materials studied in this paper, and in particular Dr. Brian Davidson for his work on developing a process for the patterning of dielectric wafers using electron beam lithography.
[1] A. Potts, D. M. Bagnall, and N. I. Zheludev, J. Opt. A, Pure Appl. Opt. 2, 193 (2004)

[2] L. R. Arnaut and L. E. Davies, in Proceedings of International Conference on Electromagnetics in Advanced Applications and Electromagnetic Waves (Nexus Media, Swanley, U.K., 1995), pp. 381-384.

[3] A. Potts, A. Papakostas, N. I. Zheludev, H. J. Coles, R. Greef, and D. M. Bagnall, Mater. Res. Soc. Symp. Proc. 722, 293 (2002).

[4] A. Papakostas, A. Potts, D. M. Bagnall, S. L. Prosvirnin, H. J. Coles, and N. I. Zheludev, Phys. Rev. Lett. 90, 107404 (2003).

[5] S. J. Elston, G. P. Bryan-Brown, and J. R. Sambles, Phys. Rev. B 44, 6393 (1991).

[6] A. V. Kats and I. S. Spevak, Phys. Rev. B 65, 195406 (2002).

[7] M. Kuwata-Gonokami, N. Saito, Y. Ino, M. Kauranen, K. Jefimovs, T. Vallius, J. Turunen, and Y. Svirko, Phys. Rev. Lett.
95, 227401 (2005).

[8] W. Zhang, A. Potts, and D. M. Bagnall, J. Opt. A, Pure Appl. Opt. 8, 878 (2006).

[9] J. Kong, Electromagnetic Wave Theory (Wiley, New York, 1986).

[10] G. D. Monteath, Applications of the Electromagnetic Reciprocity Principle (Pergamon Press, Oxford, 1973).

[11] W. Zhang, A. Potts, D. M. Bagnall, and B. R. Davidson, J. Vac. Sci. Technol. B 24, 1455 (2006).

[12] A. Potts, A. Papakostas, D. M. Bagnall, and N. I. Zheludev, Microelectron. Eng. 73-74, 367 (2004).

[13] M. Born and E. Wolf, Principles of Optics, 7th ed. (Cambridge University Press, Cambridge, 1999).

[14] S. L. Prosvirnin and N. I. Zheludev, Phys. Rev. E 71, 037603 (2005). 